\title{
THE EFFECT OF RANDOM SCALE CHANGES ON LIMITS OF INFINITESIMAL SYSTEMS
}

\author{
PATRICK L. BROCKETT \\ Department of Mathematics \\ The University of Texas \\ Austin, Texas 78712 U.S.A.
}

(Received August 25, 1977 and in revised form April 3, 1978)

ABSTRACT. Suppose $S=\left\{\left\{X_{n j}, j=1,2, \ldots, k_{n}\right\}\right\}$ is an infinitesimal system of random variables whose centered sums converge in law to a (necessarily infinitely divisible) distribution with Levy representation determined by the triple $\left(\gamma, \sigma^{2}, M\right)$. If $\left\{Y_{j}, j=1,2, \ldots\right\}$ are independent indentically distributed random variables independent of $\mathrm{S}$, then the system $S^{\prime}=\left\{\left\{Y_{j} X_{n j}, j=1,2, \ldots, k_{n}\right\}\right\}$ is obtained by randomizing the scale parameters in $S$ according to the distribution of $Y_{1}$. We give sufficient conditions on the distribution of $\mathrm{Y}$ in terms of an index of convergence of $\mathrm{S}$, to insure that centered sums from $S^{\prime}$ be convergent. If such sums converge to a distribution determined by $\left(\gamma^{\prime},\left(\sigma^{\prime}\right)^{2}, \Lambda\right)$, then the exact relationship between $\left(\gamma, \sigma^{2}, M\right)$ and $\left(\gamma^{\prime},\left(\sigma^{\prime}\right)^{2}, \Lambda\right)$ is established. Also investigated is when limit distributions from $S$ and $S^{\prime}$ are of the same type, and conditions insuring 
products of random variables belong to the domain of attraction of a stable law.

KEY WORDS AND PHRASES. General central limit theorem, products of random variables in the domain of attraction of stable laws, Lévy spectral function.

AMS (MOS) SUBJECT CLASSIFICATION (1970) CODES. 60F05, $60 F 99$.

1. INTRODUCTION AND SUMMARY.

The classical linear model for the relationship between empirical data $\mathrm{Y}$ and theoretical or "true" data $\mathrm{X}$ is to assume $\mathrm{Y}=\mathrm{X}+\varepsilon$ where $\varepsilon$ (the error) is a random variable independent of $\mathrm{X}$ with $\mathrm{E}(\varepsilon)=0$. In some cases however the error tends to depend upon $\mathrm{X}$. For example if $\mathrm{X}$ denotes the measurement of some random phenomenon we may find the empirical data agrees well with $\mathrm{X}$ for values of $\mathrm{X}$ which are small, but the error becomes increasingly greater as $\mathrm{X}$ becomes larger. Such is the case when a measuring device has a constant percentage error. If we have selected a measuring device at random from a population of devices whose constant percentage error $\mathrm{Y}$ follows the distribution function $G$, then we may model the empirical data as $Y X$ where $Y$ and $X$ are independent and $\mathrm{E}(\mathrm{Y})=1$. The empirical data can be considered as a random scale change of the theoretical data $\mathrm{x}$, or equivalently the scale parameter of $\mathrm{X}$ has been subjected to a mixture with mixing distribution function $\mathrm{G}$.

The problem we shall consider is the mathematical problem of limit distributions for sums when the scale parameter is mixed. Specifically, if $S=\left\{\left\{x_{n j}, j=1,2, \ldots, k_{n}\right\}\right\}$ is an infinitesimal system of random variables whose centered sums converge in distribution to some (infinitely divisible) random variable $X$, and if $\left\{Y_{j}, j=1,2, \ldots\right\}$ is a sequence of independent identically distributed random variables which is independent of $\mathrm{S}$, we seek conditions on the distribution of $\mathrm{Y}_{1}$ and the system $\mathrm{S}$ to insure that centered sums from the randomly scale changed system $S^{\prime}=\left\{\left\{Y_{j} X_{n j}, j=1,2, \ldots, k_{n}\right\}\right\}$ 
converge, say to $Z$. In case $S^{\prime}$ does converge, we wish to determine the exact relationship between $X$ and $Z$.

Here we take an index of convergence $\beta_{S}$ of the system $S$ and under the weak hypothesis $E\left|Y_{1}\right|^{\beta} S^{+\delta}<\infty$ for some $\delta>0$, we obtain a necessary and sufficient condition for convergence of the system $S^{\prime}$. When $S^{\prime}$ converges we then obtain the exact relationship between the limit distributions of $X$ and $Z$. These results are then applied to a most commonly occurring system, namely that consisting of normed random variables whose distribution is attracted to stable laws with exponent $\alpha$. In this case we find $\beta_{S}=\alpha$. In the classical central limit theorem $(\alpha=2)$ we find that if $\mathrm{EY}^{2}<\infty$, then $\mathrm{X}$ is attracted to the normal distribution if and only if $\mathrm{XY}$ is attracted to the normal distribution, and moreover the same norming constants work. For $\alpha<2$ we find that if $E\left|Y_{1}\right|^{a+\delta}<\infty$ and $X$ is attracted to a stable of index $\alpha$, then $X Y$ is attracted to the same stable law, the same norming constants work, and the exact scale change between the two resulting stable laws in calculated. We are also interested in when the limit laws of $S$ and $S^{\prime}$ are of the same type. A necessary and sufficient condition for this to happen is that the 1 imit 1 aw be either of purely stable type, or a mixture of stable and normal type.

\section{PREL DII NARIES - - AN INDEX OF CONVERGENCE}

Let us recall several important facts from [3].

If $S=\left\{\left\{x_{n j}, j=1,2, \ldots, k_{n}\right\}\right\}$ is an infinitesimal system of random variables, the functions $M_{n}$ are defined by

$$
M_{n}(x)= \begin{cases}\sum_{j=1}^{k} F_{X} \quad(x) & \text { for } x<0 \\ \sum_{j=1}^{k}\left\{F_{n, j}(x)-1\right\} & \text { for } x>0\end{cases}
$$


where $F_{n, j}$ is the distribution function of $X_{n j}$.

We shall say that the system $S$ is convergent to $X$ if there exists a sequence of real numbers $\left\{C_{n} n=1,2, \ldots\right\}$ such that $\sum_{j=1}^{k} x_{n, j}-C_{n}$ converges in distribution to $x$ as $n \rightarrow \infty$. In this case $x$ is infinitely divisible with characteristic function whose logarithm is of the form

$$
\log f_{X}(u)=i u \propto-\frac{\sigma^{2} u^{2}}{2}+\int_{-x}^{x}\left\{e^{i u x}-1-\frac{i u x}{1+x^{2}}\right\} d M(x) .
$$

We shall simply write $\mathrm{X} \sim\left(\alpha, \sigma^{2}, \mathrm{M}\right)$ to express the fact that $\mathrm{X}$ has such $\mathrm{a}$ characteristic function. The symbol $\int_{-c}^{d}$ represents $\lim \left\{\int_{a}^{d}+\int_{-c}^{b}: a \downarrow 0\right.$ and $\mathrm{b} \uparrow 0\}$. All integrals are taken in the Lebesgue-Stieltjes sense. The set of points of continuity of a function $g$ will be denoted Cont $(g)$. Discont(g) is defined to be the set of points at which $g$ is not continuous. We shall hence forth use $\Sigma$ to denote $\sum_{j=1}^{k}$ and $F_{n j}$ to denote $F_{X_{n j}}$.

DEF INITION. Let $S$ be an infinitesimal system. The index for for the system $S$ is defined by

$$
\beta_{S}=\inf \left\{\delta>0: \lim _{(x, n) \rightarrow(0, \infty)}|x|^{\delta} \int_{|t|>|x|} d_{n}(t)=0\right\}
$$

with $\beta_{S}=\infty$ if no such $\delta$ above exists.

One should remark here that the index $\beta_{S}$ as defined above has some relation to an index defined in 1961 by Blumenthal and Getoor [2] and later generalized by Berman (1965). They define the index $\beta^{\mathrm{M}}$ for the Levy spectral function $M$ by

$$
\begin{aligned}
& \beta^{M}=\inf \left\{c>0: \int_{-1}^{1}|x|^{c} d M(x)<\infty\right\}, \text { and proved } \\
& \beta^{M}=\inf \left\{c>0: \lim _{x \rightarrow 0}\left\{|x|^{c}(M(-|x|)-M(|x|)\}=0\right\} .\right.
\end{aligned}
$$


As a similar result in [4] it was shown that then $S$ converges to $X \sim\left(\gamma, \sigma^{2}, M\right)$ we have for a $\in$ Cont $(M)$

$$
\beta_{S}=\inf \left\{\varepsilon \geq B^{M}: \int_{|x|<a}|x|^{\varepsilon} \mathrm{dM}_{n}(x) \rightarrow \int_{|x|<a}|x|^{\varepsilon} \mathrm{dM}(\mathrm{x})\right\}
$$

with $\beta_{S}=\infty$ if the above set is empty. An easy way of calculating $\beta_{S}$ is then given by noting that

$$
\begin{aligned}
& \lim _{\varepsilon \rightarrow 0} \lim _{n \rightarrow \infty} \sup _{|x|<\varepsilon}|x|^{\gamma} d_{n}(x)=0 \text { if } \gamma>\beta_{S} \\
& \lim _{n \rightarrow \infty} \int_{|x|<\varepsilon}|x|^{\gamma} d_{n}(x)=\infty \text { for any } \varepsilon>0 \text { if } \gamma<\beta_{S} .
\end{aligned}
$$

We next recall a variational sum result proved in [4].

THEOREM 1. Suppose $S$ is an infinitesimal system converging to $X \sim\left(\alpha, \sigma^{2}, M\right)$, and suppose that $g$ is a bounded function which satisfies $g(x)=0\left(|x|^{\gamma}\right)$ as $x \rightarrow 0$ for some $\gamma>\beta_{S}$ and which is either continuous or is of bounded variation over $(-\infty, 0)$ and over $(0, \infty)$ with discont $(g) \cap$ discont $(M)=\emptyset . \quad$ Then we have

$$
\lim _{n \rightarrow \infty} \sum E\left(g\left(X_{n j}\right)\right)=\lim _{n \rightarrow x} \int_{(-\infty, x)} g(x) d M_{n}(x)=\int_{(-x, x)} g(x) d M(x)
$$

REMARK 1. Some comments about the index $\beta_{S}$ are in order. If the system $S$ is convergent to $X \sim\left(\sigma, \sigma^{2}, M\right)$, then $F^{M} \leq F_{S} \leq \infty$ and either of the two equalities is possible. For an example where $\beta_{S}=6^{M}$, see [4] where it is shown that if $\left\{x_{1}, x_{2}, \ldots\right\}$ is a sequence of independent identically distributed random variables belonging to the domain of attraction of a stable distribution with exponent $\alpha$, and if $S=\left\{\left\{x_{j} / B_{n}, j=1,2, \ldots, n\right\}\right\}$ 
then $\beta_{S}=\alpha$. It is clear that in this case $\beta^{M}=\left\{\begin{array}{ll}\alpha & \text { if } \alpha<2 \\ 0 & \text { if } \alpha=2\end{array}\right.$. In [3] an example is given showing $\beta_{S}$ can be anything. Namely for $\gamma \leq \infty$ a convergent system $S$ is found such that $\beta_{S}=\lambda$. To show that $\beta_{S}$ measures "how" the system converges rather than to what it converges, an example in [3] is given showing that for any Levy spectral function $M$ and for any $\lambda \geq \beta^{M}$ there exists a system $S$ converging to $(0,0, M)$ with $\beta_{S}=\lambda$. The index $\beta_{S}$ has proved to be the appropriate index for studying variational sums of infinitesimal systems [4], and has been shown to be an extension of the Blumenthal-Getoor index for stochastic processes with independent increments [5] which allows a unified treatment of variational sums of such processes. Let us also state for reference the general limit theorem as found in Gnedenko and Kolmogorov (1968) or Tucker (1967).

THEOREM 2. Suppose $S=\left\{\left\{x_{n j}: j=1,2, \ldots, k_{n}\right\}\right\}$ is an infinitesimal system. A necessary and sufficient condition that $\Sigma x_{n j}-c_{n} \rightarrow X \sim\left(\alpha, \sigma^{2}, M\right)$ is that the following three conditions all hold
A) $M_{n}(x) \rightarrow M(x)$ for all $x \in \operatorname{Cont}(M)$
B) $\lim _{\varepsilon \rightarrow 0}\left\{1\right.$ im $\sup \{\}_{n \rightarrow \infty}\left\{\int_{|x|<\varepsilon} x^{2} d M_{n}(x)-\Sigma\left(\int_{|x|<\varepsilon} x d x_{n j}(x)\right)^{2}\right\}=\sigma^{2}$
C) $\int_{|x|<\tau} x d M_{n}(x)-c_{n} \rightarrow a+\int_{|x|<\tau} x^{3} /\left(1+x^{2}\right) d M(x)-\int_{|x|<T} x /\left(1+x^{2}\right) d M(x)$

for any $\tau>0$.

\section{A LIMIT THEOREM FOR SCALE MIXED SYSTEMS AND APPLICATIONS}

We sha11 now proceed to answer the questions posed in the introduction. Namely when $S$ is convergent, the index $\beta_{S}$ yields the needed tool for obtaining conditions insuring $S^{\prime}$ is convergent. The precise formulation, and the 
exact relationship between the limit 1 aws of $S$ and of $S^{\prime}$ is given in the following theorem. Also to be noted is that the convergence properties of $S^{\prime}$ are the same as that of $S$ (i.e., $\beta_{S}=\beta_{S^{\prime}}$ ) and the behavior of the limiting Levy functions are the same $\left(\beta^{M}=\beta^{\Lambda}\right)$. In particular since an infinitely divisible distribution is continuous if and only if the corresponding Levy spectral function is unbounded, it follows that the limit distributions of $S$ and $S^{\prime}$ are simultaneous continuous or not continuous.

THEOREM 3. Suppose that $S=\left\{\left(x_{n j}, j=1,2, \ldots, k_{n}\right\}\right\}$ is convergent to $X \sim\left(\gamma, \sigma^{2}, M\right)$ i.e., $\Sigma X_{n j}-c_{n} \stackrel{d}{\longrightarrow} X . \quad$ Let $\left\{Y_{j}, j=1,2, \ldots\right\}$ be a sequence of independent identically distributed random variables with non-degenerate common distribution function $F_{Y}$ and with $E|Y|^{\beta S^{+\kappa}}<\infty$ for some $\kappa>0$. Assume also that $\left\{Y_{j}, j=1,2, \ldots\right\}$ is independent of the system $S$. Then a necessary and sufficient condition that $S^{\prime}=\left\{\left\{Y_{j} X_{n j}, j=1,2, \ldots, k_{n}\right\}\right.$ $\mathrm{n}=1,2, \ldots\}$ be convergent is

$$
\begin{aligned}
C_{0} & =\lim _{\varepsilon \rightarrow 0} \lim _{n \rightarrow \infty} \sup \int_{|x|<\varepsilon} x^{2} d M_{n}(x) \\
& =\lim _{\varepsilon \rightarrow 0} \lim _{n \rightarrow \infty} \inf \int_{|x|<\varepsilon} x^{2} d M_{n}(x)
\end{aligned}
$$

exists and be finite (so that by Remark 1 necessarily $\left.\beta_{S} \leq 2\right)$. If $S^{\prime}$ converges to $Z \sim\left(\gamma^{\prime},\left(\sigma^{\prime}\right)^{2}, \Lambda\right)$, then the following are true:

$1^{0}$ The centering constants for $S^{\prime}$ can be chosen as

$$
\begin{aligned}
d_{n} & =\Sigma \int_{|x|<\tau} x d F_{X_{n j} Y_{j}}(x)-\gamma^{\prime}-\Sigma \int_{|x|<\tau} x^{3} /\left(1+x^{2}\right) d F X_{n j} Y_{j}(x) \\
& +\Sigma \int_{|x|>\tau} x /\left(1+x^{2}\right) d F X_{n j} Y_{j}(x)
\end{aligned}
$$


$2^{0}$

$$
\Lambda(x)= \begin{cases}\int_{(-\infty, 0)}\left\{1-F_{Y}(x / t)\right\} d M(t)+\int_{(0, \infty)} F_{Y}(x / t) d M(t) & \text { for } x<0 \\ -\int_{(-\infty, 0)} F_{Y}(x / t) d M(t)+\int_{(0, \infty)}\left\{F_{Y}(x / t)-1\right\} d M(t) & \text { for } x>0\end{cases}
$$

$3^{0}$

$$
\left(\sigma^{\prime}\right)^{2}=C_{0} \operatorname{Var}(Y)+\sigma^{2}(E(Y))^{2}
$$

where $\mathrm{C}_{0}$ is as defined in (3.1)

$$
\begin{array}{ll}
4^{0} & \beta_{S}=\beta_{S^{\prime}} \\
5^{0} & \beta^{\Lambda}=\beta^{M} .
\end{array}
$$

NOTE: 1. If $\beta_{S}<2$ then (3.1) is automatically satisfied with $C_{0}=0$ by (2.3) and necessarily $\sigma^{2}=0$ (otherwise $\beta_{S} \geq 2$ ), so $3^{0}$ says $\left(\sigma^{\prime}\right)^{2}=0$ in this case. Also when $\beta_{S}=2$ and (3.1) holds we need only assume $E\left(Y^{2}\right)<\infty$ to obtain the conclusion.

2. It should be noted that the mere meaningfulness of formula $2^{0}$ may not be sufficient for the conclusion of the theorem. Examples of this are given in [3].

PROOF. 1) We first note that $S^{\prime}$ is indeed an infinitesimal system. Now, an easy calculation yields

$$
\begin{aligned}
F_{X_{n j} Y j}(x)=\int_{(-\infty, 0)}\left\{1-F_{Y}(x / t) d F_{n j}(t)\right. & +\int_{(0, \infty)} F_{Y}(x / t) d F{ }_{n j}(t) \\
& +P\left[X_{n j}=0\right] X_{[0, \infty)}(x) .
\end{aligned}
$$


Here $\chi_{A}(x)=1$ if $x \in A$ and 0 if $x \notin A$. Let

$x \in A$ and 0 if $x \notin A$. Let

and let

$$
\Lambda_{n}(x)= \begin{cases}\sum F_{X_{n j} Y_{j}}(x) & \text { for } x<0 \\ \sum\left\{F_{X_{n j} Y_{j}}(x)-1\right\} & \text { for } x>0\end{cases}
$$

$$
g(x)= \begin{cases}1-F_{Y}(x) & x>0 \\ F_{Y}(x) & x<0 .\end{cases}
$$

Then using (3.2) in (3.3) we have for $x<0$

$$
\begin{aligned}
\Lambda_{n}(x) & =\int_{(-\infty, 0)}\left\{1-F_{Y}(x / t)\right\} d M_{n}(t)+\int_{(0, \infty)} F_{Y}(x / t) d M_{n}(t) \\
& =\int_{(-\infty, \infty)} g(x / t) d M_{n}(t) .
\end{aligned}
$$

while for $x>0$ we have

$$
\begin{aligned}
\Lambda_{n}(x) & =\Sigma\left\{-\int_{(-\infty, 0)} g(x / t) d F_{n j}(t)-\int_{(0, \infty)} g(x / t) d F_{n j}(t)\right\} \\
& =-\int_{(-\infty, \infty)} g(x / t) d M_{n}(t) .
\end{aligned}
$$

Thus we have

$$
\Lambda_{n}(x)= \begin{cases}\int_{(-\infty, \infty)} g(x / t) d M_{n}(t) & \text { for } x<0 \\ -\int_{(-\infty, \infty)} g(x / t) d M_{n}(t) & \text { for } x>0 .\end{cases}
$$

2) In this part of the proof we shall show that if $\mathrm{E}|\mathrm{Y}|^{\beta} \mathrm{S}^{+\delta}<\infty$, then we always have $\lim _{n \rightarrow \infty} \Lambda_{n}(x)=\Lambda(x)$ for all $x \in \operatorname{Cont}(\Lambda)$. Indeed since $E|Y|^{\beta} S^{+\delta}<\infty$ we have $\left\{1-F_{Y}(t)+F_{Y}(-t)\right\}=0\left(t^{-\beta} S^{-\delta}\right)$ as $t \rightarrow \infty$. By the defi- 
nition of $g$, we can thus see that for fixed $x, g(x / t)=0\left(|t|^{\beta} s^{+\delta}\right.$ ) as $t \rightarrow 0$. Let us write $g_{x}(t)=g(x / t)$. By Theorem 1 we have

$$
\lim _{n \rightarrow \infty} \int_{(-\infty, \infty)} g_{x}(t) d M_{n}(t)=\int_{(-\infty, \infty)} g_{x}(t) d M(t)
$$

for every $x$ such that $\operatorname{discont}\left(g_{x}\right) \cap \operatorname{discont}(M)=\emptyset$. Thus using (3.5), (3.4) and $2^{0}$ we have $\lim _{n \rightarrow \infty} \Lambda_{n}(x)=\Lambda(x)$ for all $x$ such that discont $\left(g_{x}\right) \cap$ discont $(M)=\emptyset$. Let $U=\left\{x: \operatorname{discont}\left(g_{x}\right) \cap \operatorname{discont}(M)=\emptyset\right\}$. It remains to show Cont $(\Lambda) \subseteq \mathcal{U}$. Equivalently we show $\mathcal{U}^{c} \subseteq$ discont $(\Lambda)$. If $\mathrm{y}_{0} \in \mathcal{U}^{c}$ then there exists a $t_{0}$ such that $g_{y_{0}}$ and $M$ are both discontinuous at $t_{0}$. For simplicity let us assume that $y_{0}>0$ and $t_{0}>0$. A similar argument can be used in any other case. Thus we have $g_{y_{0}}\left(t_{0}+0\right)-g_{y_{0}}\left(t_{0}-0\right)>0$ and $d M\left(\left\{t_{0}\right\}\right)>0$, so that for any small $h>0$ we have

$$
\begin{aligned}
\Lambda\left(y_{0}\right. & +h)-\Lambda\left(y_{0}-h\right) \geq\left\{F_{Y}\left(\frac{y_{0}+h}{t_{0}}\right)-F_{Y}\left(\frac{y_{0}-h}{t_{0}}\right)\right\} d M\left(\left\{t_{0}\right\}\right) \\
& =\left\{F_{Y}\left(\frac{y_{0}}{t_{0}-\eta}\right)-F_{Y}\left(\frac{y_{0}}{t_{0}+\beta}\right)\right\} d M\left(\left\{t_{0}\right\}\right)=\left\{g_{y_{0}}\left(t_{0}+\beta\right)-g_{y_{0}}\left(t_{0}-\eta\right)\right\} d M\left(\left\{t_{0}\right\}\right) \\
& \geq\left\{g_{y_{0}}\left(t_{0}+0\right)-g_{y_{0}}\left(t_{0}-0\right)\right\} d M\left(\left\{t_{0}\right\}\right)=b>0
\end{aligned}
$$

where $\beta=t_{0} h /\left(y_{0}-h\right)$ and $\eta=t_{0} h / y_{0}+h$. Since $\Lambda\left(y_{0}+h\right)-\Lambda\left(y_{0}-h\right) \geq b>0$ for all h, we must have $y_{0} \in \operatorname{discont}(\Lambda)$. Thus $\Lambda_{n}(x) \rightarrow \Lambda(x)$ for all $x \in \operatorname{Cont}(\Lambda)$. To see that $\Lambda$ is a Levy spectral function, noted that $\Lambda$ is monotone increasing on $(-\infty, 0)$ and on $(0, \infty)$ and $\Lambda(+\infty)=\Lambda(-\infty)=0$. We must also show that $\int_{(-1,1)} x^{2} d \Lambda(x)<\infty$. The proof of this is accomplished in part 5) below.

3) In this part of the proof we shall determine how to calculate integrals of the form $\int_{(-\infty, \infty)} f(x) d \Lambda_{n}(x)$ and $\int_{(-\infty, \infty)} f(x) d \Lambda(x)$ where $\Lambda_{n}$ 
is given by (3.4) and $\Lambda$ is given by $2^{0}$. Now for $0<a<b<\infty$, let $\chi_{(a, b]}$ denote the indicator function of the interval $(a, b]$ and calculate

$$
\begin{aligned}
\Lambda_{n}(b)-\Lambda_{n}(a) & =\int_{(-\infty, \infty)} \int_{(-\infty, \infty)} \chi_{\frac{1}{t}(a, b]}(u) d F_{Y}(u) d M_{n}(t) \\
& =\int_{(-\infty, \infty)} \int_{(-\infty, \infty)} \chi_{(a, b]}(t u) d F_{Y}(u) d M_{n}(t)
\end{aligned}
$$

so that

$$
\int_{(-\infty, \infty)} f(x) d \Lambda_{n}(x)=\int_{(-\infty, \infty)} \int_{(-\infty, \infty)} f(u x) d F_{Y}(u) d M_{n}(x)
$$

holds when $f$ is the indicator function of an interval in $(-\infty, \infty)$. Standard approximation techniques now allow us to conclude (3.6) holds for the function in question. Also (3.6) holds if we replace $\Lambda_{n}$ by $\Lambda$ and $M_{n}$ by $M$.

4) In this part of the proof we show that $4^{0}$ holds. We shall use (2.4) to show $\beta_{S}=\beta_{S}$. . Now for any $\delta>0$ we have

$$
\begin{aligned}
& \int_{|x|<\varepsilon}|x|^{\delta} d \Lambda_{n}(x)=\int_{(-\infty, \infty)}|x|^{\delta} \int_{(-\infty, \infty)}|u|^{\delta} \chi_{(-\varepsilon, \varepsilon)}(u x) d F_{Y}(u) d M_{n}(x) \\
& =\int_{|x|<1}|x|^{\delta} \int_{|u x|<\varepsilon}|u|^{\delta} d_{Y}(u) d M_{n}(x)+\int_{|x| \geq 1}|x|^{\delta} \int_{|u x|<\varepsilon}|u|^{\delta} d_{Y}(u) d M_{n}(x) .
\end{aligned}
$$

However concerning the second term above we see that

$$
\lim _{\varepsilon \rightarrow 0} \underset{n \rightarrow \infty}{\lim \sup _{n \rightarrow \geq 1}} \int_{|x|}|x|_{|u x|<\varepsilon}|u|^{\delta} d_{Y}(u) d M_{n}(x)=0 .
$$


Thus to show $\beta_{S}=\beta_{S}$, we must only show

$\lim _{\varepsilon \rightarrow 0} \lim _{n \rightarrow \infty} \int_{|x|<1}|x|^{\delta} \int_{|u x|<\varepsilon}|u|^{\delta} d_{Y}(u) d M_{n}(x)=0$ if $\delta>\beta_{S}$ and $\lim _{n \rightarrow \infty} \inf _{|x|<1}|x|^{\delta} \int_{|u x|<\varepsilon}|u|^{\delta} d_{Y}(u) d M_{n}(x)=\infty$ for any $\varepsilon>0$ if $\delta<\beta_{S}$. Suppose then that $\delta<\beta_{S}$ and choose $\varepsilon_{1}$ so that $\int_{|x|<\varepsilon_{1}}|x|^{\delta} d F_{Y}(x)>0$. Now $\int_{|x|<1} \int_{|u x|<\varepsilon}|u x|^{\delta} d_{Y}(u) d M_{n}(x) \geq \int_{|x|<1}|x|^{\delta} \int_{|u|<\varepsilon}|u|^{\delta} d_{Y}(u) d M_{n}(x)$ so that $\lim _{\mathrm{n} \rightarrow \infty} \inf _{|\mathbf{x}|<1} \int_{|\mathrm{xu}|<\varepsilon_{1}}|\mathrm{ux}|^{\delta} \mathrm{dF}_{\mathrm{Y}}(\mathrm{u}) \mathrm{dM}_{\mathrm{n}}(\mathrm{x}) \geq$ $\int_{|u|<\varepsilon_{1}}|u|^{\delta} \mathrm{dF}_{\mathrm{Y}}(\mathrm{u}) \lim _{\mathrm{n} \rightarrow \infty} \inf _{|\mathrm{x}|<1}|\mathbf{x}|^{\delta} \mathrm{dM}_{\mathrm{n}}(\mathrm{x})=\infty$, and thus $\beta_{\mathrm{S}} \leq \beta_{\mathrm{S}^{\prime}}$. To see that $\beta_{S} \geq \beta_{S}$, let $\gamma>\beta_{S}$ be such that $E|Y|^{\gamma}<\infty$ and pick $\alpha>0$ such that $\delta-\gamma<\alpha<\delta-\beta_{S}$ i.e., such that $\beta_{S}<\delta-\alpha<\gamma$. Then $\mathrm{E}|\mathrm{Y}|^{\delta-\alpha}<\infty$ and also $\int_{|x|<1}|x|^{\delta-\alpha} d_{n}(x)$ is bounded in $n$. Now $\int_{|x|<1} \int_{|u x|<\varepsilon}|u x|^{\delta} d F_{Y}(u) d M_{n}(x) \leq$ $\varepsilon^{\alpha} E|Y|^{\delta-\alpha} \int_{|x|<1}|x|^{\delta-\alpha} d M_{n}(x)$. Thus

$\lim _{\varepsilon \rightarrow 0} \lim _{n \rightarrow \infty} \int_{|x|<1} \int_{|u x|<\varepsilon}|u x|^{\delta} d_{F_{Y}}(u) d M_{n}(x)=0$. I.e. $\beta_{S^{\prime}} \leq \beta_{S}$ and hence $\beta_{S^{\prime}}=\beta_{S}$.

5) In this part of the proof we shall show $5^{0}$ holds by showing

$$
\int_{|x|<1}|x|^{\delta} d \Lambda(x)=\left\{\begin{array}{lll}
<\infty & \text { if } & \delta>\beta^{M} \\
\infty & \text { if } & \delta<\beta^{M}
\end{array}\right.
$$

Suppose $\delta \geq \beta^{M}$ and that $\int_{(-1,1)}|x|^{\delta} \mathrm{dM}(x)<\infty$. Now we know by hypothesis that there is some $\gamma \geq \beta_{S} \geq \beta^{M}$ such that $E|Y|^{\gamma}<\infty$. Without loss of generality we may assume $\delta \leq \gamma$ so that we have 


$$
\begin{aligned}
& \int_{|\mathbf{x}|<1}|\mathbf{x}|^{\delta} \mathrm{d} \Lambda(\mathrm{x})=\int_{|\mathbf{x}|<1}|\mathrm{x}|^{\delta} \int_{|\mathrm{ux}|<1}|\mathrm{u}|^{\delta} \mathrm{dF}_{\mathrm{Y}}(\mathrm{u}) \mathrm{dM}(\mathrm{x}) \\
& \quad+\int_{|\mathrm{x}| \geq 1}|\mathrm{x}|^{\delta} \int_{|\mathrm{ux}|<1}|\mathrm{u}|^{\delta} \mathrm{dF}_{\mathrm{Y}}(\mathrm{u}) \mathrm{dM}(\mathrm{x}) \leq \mathrm{E}|\mathrm{Y}|^{\delta} \int_{|\mathbf{x}|<1}|\mathbf{x}|^{\delta} \mathrm{dM}(\mathrm{x})+\mathrm{M}(-1)-\mathrm{M}(1)<\infty
\end{aligned}
$$

i.e. $\beta^{\Lambda} \leq \beta^{M}$. On the other hand, if $\delta<\beta^{M}$ so that $\int_{|\mathbf{x}|<1}|\mathbf{x}|^{\delta} \mathrm{dM}(\mathbf{x})=\infty$, then choosing $1 \geq c>0$ such that $P\left[Y<\frac{1}{c}\right]>0$ we have

$$
\begin{aligned}
& \int_{|x|<1}|\mathrm{x}|^{\delta} \mathrm{d} \Lambda(\mathrm{x}) \geq \int_{|\mathrm{x}|<\mathrm{c}}|\mathrm{x}|^{\delta} \int_{|\mathrm{ux}|<1}|\mathrm{u}|^{\delta} \mathrm{dF}_{\mathrm{Y}}(\mathrm{u}) \mathrm{dM}(\mathrm{x}) \\
& \geq\left\{\int_{|\mathrm{x}|<\mathrm{c}}|\mathrm{x}|^{\delta} \mathrm{dM}(\mathrm{x})\right\}\left\{\int_{|\mathrm{u}|<\frac{1}{\mathrm{c}}}|\mathrm{u}|^{\delta} \mathrm{dF}_{\mathrm{Y}}(\mathrm{u})\right\}=\infty .
\end{aligned}
$$

Thus $\beta^{\Lambda} \geq \beta^{M}$ and hence $\beta^{\Lambda}=\beta^{M}$. In particular, choosing $\delta=2$ we see $\Lambda$ is indeed a Levy spectral function.

6) An elementary calculation using the Helly Bray theorem and Theorem 2 shows that the centering can be taken as in $1^{0}$ when (3.1) holds.

7) In this part of the proof we shall show that the finiteness of the limit in (3.1) is necessary. We shall show that if

$$
\lim _{n \rightarrow \infty} \sup _{|x|<\varepsilon} x^{2} d M_{n}(x)=\infty
$$

then

$$
\lim _{n \rightarrow \infty} \sup _{n \rightarrow \infty}\left\{\int_{|x|<\varepsilon} x^{2} d \Lambda_{n}(x)-\Sigma\left(\int_{|x|<\varepsilon} x d F_{X_{n j} Y}(x)\right)^{2}\right\}=\infty .
$$


Hence by the general 1 imit theorem $S^{\prime}$ cannot be convergent. Let $\varepsilon>1$ and for simplicity of notation let us write

$$
\sigma_{n}^{2}(\varepsilon)=\int_{|x|<\varepsilon} x^{2} d \Lambda_{n}(x)-\Sigma\left(\int_{|x|<\varepsilon} x d F X_{n j} y_{j}(x)\right)^{2}
$$

Then

$$
\begin{aligned}
& \sigma_{n}^{2}(\varepsilon) \geq \int_{|x|<\varepsilon} x^{2} \int_{|u x|<\varepsilon} u^{2} d F_{Y}(u) d M_{n}(x) \\
& -\Sigma\left\{\int_{|x|<\varepsilon} x \int_{|u x|<\varepsilon} u d F_{Y}(u) d F_{n j}(x)\right\}^{2} \\
& -2 \Sigma\left\{\int_{|x|<\varepsilon}{ }^{x} \int_{|u x|<\varepsilon} u d F_{Y}(u) d F_{n j}(x)\right\}\left\{\int_{|x| \geq \varepsilon} x \int_{|u x|<\varepsilon} u d F_{Y}(u) d F_{n j}(x)\right\} \\
& -\Sigma\left\{\int_{|x| \geq \varepsilon} x \int_{|u x|<\varepsilon} u d F_{Y}(u) d F_{n j}(x)\right\}^{2} \text {. }
\end{aligned}
$$

CLAIM 1. $\quad \lim \sup _{n \rightarrow \infty} \Sigma\left\{\int_{|x| \geq \varepsilon} x \int_{|u x|<\varepsilon} u d F_{Y}(u) d F_{n j}(x)\right\}^{2}=0$.

PROOF. By the Schwartz inequality,

$$
\begin{aligned}
&\left(\int_{|x| \geq \varepsilon}\left\{x \int_{|u x|<\varepsilon} u d F_{Y}(u)\right\} d F_{n j}(x)\right)^{2} \\
& \leq\left(\int_{|x| \geq \varepsilon}\left\{x \int_{|u x|<\varepsilon} u d F_{Y}(u)\right\}^{2} d F_{n j}(x)\right) P\left[\left|x_{n j}\right| \geq \varepsilon^{2}\right] \\
& \leq \max _{j \leq j \leq k} P\left[\left|x_{n j}\right| \geq \varepsilon^{2}\right] \int_{|x| \geq \varepsilon^{2}} x^{2} \int_{|u x|<\varepsilon} u^{2} d F_{Y}(u) d F_{n j}(x) .
\end{aligned}
$$


Using the infinitesimality of the system, we have the first term converging to zero. The sum of the second terms is bounded, since by the Helly-Bray theorem

$$
\begin{aligned}
& \lim \sup _{n \rightarrow \infty} \int_{|x| \geq \varepsilon} x^{2} \int_{|u x|<\varepsilon} u^{2} d F_{Y}(u) d M_{n}(x) \\
& \quad \leq\left\{E\left(Y^{2}\right) \int_{\varepsilon^{2}<|x|<\varepsilon} x^{2} d M(x)+\varepsilon^{2} \int_{|x| \geq \varepsilon} d M(x)\right\} .
\end{aligned}
$$

This completes the proof of Claim 1 . Note that using the above we actually have

$$
\lim _{\varepsilon \rightarrow 0} \lim _{n \rightarrow \infty} \sup _{|x| \geq \varepsilon} x^{2} \int_{|u x|<\varepsilon} u^{2} d F_{Y}(u) d M_{n}(x)=0
$$

CLAIM 2 .

$$
\begin{aligned}
& \left|\Sigma\left\{\int_{|x|<\varepsilon} 2^{x} \int_{|u x|<\varepsilon} u d F_{Y}(u) d F_{n j}(x)\right\}\left[\int_{|x| \geq \varepsilon^{2}} x \int_{|u x|<\varepsilon} u d F_{Y}(u) d F_{n j}(x)\right\}\right| \\
& \leq\left\{E\left(Y^{2}\right)\right\}^{\frac{3}{2}} \mathrm{o}(1)\left\{\int_{|x|<\varepsilon} 2^{\left.x^{2} d M_{n}(x)\right\}^{\frac{1}{2}}}\right. \text {. }
\end{aligned}
$$

PROOF. By the Schwarz inequality for sums, the sum in the claim is no larger than

$$
\left\{\Sigma\left(\int_{|x|<\varepsilon} 2^{x} \int_{|u x|<\varepsilon} u d F_{Y}(u) d F_{n j}(x)\right)^{2}\right\}^{\frac{1}{2}}\left\{\Sigma\left(\int_{|x| \geq \varepsilon} x \int_{|u x|<\varepsilon} u d F Y(u) d F_{n j}(x)\right)^{2}\right\}^{\frac{1}{2}} .
$$


Applying Claim 1 to the second term and the Schwarz inequality to the first term in the above product we have that the left hand side does not exceed

$$
\left\{\int_{|x|<\varepsilon} x^{2}\left(\int_{|u x|<\varepsilon} u d F_{Y}(u)\right)^{2} d M_{n}(x)\right\}^{\frac{1}{2}} o(1) \leq\left\{E\left(Y^{2}\right)\right\}^{\frac{1}{2}} o(1)\left\{\int_{|x|<\varepsilon} x^{2} d M_{n}(x)\right\}^{\frac{1}{2}}
$$

which completes the proof of Claim 2.

Now using the inequality

$$
\begin{aligned}
& \Sigma\left(\int_{|x|<\varepsilon}{ }^{2} \int_{|u x|<\varepsilon} u d F_{Y}(u) d F_{n j}(x)\right\}^{2} \\
& \quad \leq \int_{|x|<\varepsilon} x^{2}\left\{\int_{|u x|<\varepsilon} u d F_{Y}(u)\right\}^{2} d M_{n}(x)
\end{aligned}
$$

and using Claims 1 and 2 in (3.9) yields

$$
\begin{aligned}
\sigma_{n}^{2}(\varepsilon) & \geq \int_{|x|<\varepsilon} x^{2}\left\{\int_{|u x|<\varepsilon} u^{2} d F_{Y}(u)-\left(\int_{|u x|<\varepsilon} u d F_{Y}(u)\right)^{2}\right\} d M_{n}(x) \\
& -2\left(E\left(Y^{2}\right)\right)^{\frac{3}{2}} o(1)\left\{\int_{|x|<\varepsilon} 2^{\left.x^{2} d M_{n}(x)\right\}^{\frac{1}{2}}+o(1)}\right.
\end{aligned}
$$

Since (3.7) implies $\beta_{S} \geq 2$, we know $E\left(Y^{2}\right)<\infty$. Let $\delta>0$ be chosen such that $\operatorname{Var}(\mathrm{Y})-\delta>0$ and choose $\varepsilon_{0}^{2}>0$ so small that if $|\mathbf{x}|<\varepsilon_{0}^{2}$, then

$$
\operatorname{Var}(\mathrm{Y})-\delta \leq \int_{|\mathrm{ux}|<\varepsilon_{0}} \mathrm{u}^{2} \mathrm{dF} \mathrm{Y}_{\mathrm{Y}}(\mathrm{u})-\left(\int_{|\mathrm{ux}|<\varepsilon_{0}} \mathrm{udF}_{\mathrm{Y}}(\mathrm{u})\right)^{2} \leq \operatorname{Var}(\mathrm{Y})+\delta
$$


Then for $\varepsilon \leq \varepsilon_{0}$ in (3.11) we have

$$
\begin{aligned}
& \sigma_{n}^{2}(\varepsilon) \geq\{\operatorname{Var}(Y)-\delta\} \int_{|x|<\varepsilon} 2^{x^{2} d M_{n}(x)-2 E\left(Y^{2}\right) o(1)\left\{\int_{|x|<\varepsilon^{2}} x^{2} d M_{n}(x)\right\}^{\frac{1}{2}}+o(1)} \\
& =\left\{\int_{|x|<\varepsilon} 2^{\left.x^{2} d M_{n}(x)\right\}^{\frac{1}{2}}\left\{(\operatorname{Var}(Y)-\delta)\left(\int_{|x|<\varepsilon} x^{2} d M_{n}(x)\right)^{\frac{3}{2}}-2 E\left(Y^{2}\right) o(1)\right\}+o(1) .}\right.
\end{aligned}
$$

By (3.7) the $1 \mathrm{im}$ sup as $\mathrm{n} \rightarrow \infty$ of the right hand side of (3.12) is $+\infty$, i.e., $\lim _{n \rightarrow \infty} \sup _{n}^{2}(\varepsilon)=\infty$, and $S^{\prime}$ cannot be convergent.

8) In this part of the proof we show that if (3.1) holds, then $S^{\prime}$ is convergent to $\mathrm{x} \sim\left(\alpha^{\prime},\left(\sigma^{\prime}\right)^{2}, \bar{\Lambda}\right)$. Since we have al ready shown that $\lim _{n \rightarrow \infty} \Lambda_{n}(x)=\Lambda(x)$ for all $x \in \operatorname{Cont}(\Lambda)$, to obtain this part of the proof, we need only show that with $\sigma_{n}^{2}(\varepsilon)$ as given we have

$$
\lim _{\varepsilon \rightarrow 0} \underset{n \rightarrow \infty}{\lim \sup _{n}} \sigma_{n}^{2}(\varepsilon)=\lim _{\varepsilon \rightarrow 0} \underset{n \rightarrow \infty}{\lim \inf } \sigma_{n}^{2}(\varepsilon)=\left(\sigma^{\prime}\right)^{2}
$$

Now, if $\beta_{S}<2$, then by $(2.3)$ we must have $\sigma^{2}=0$, and $C_{0}=0$. However by part 5 of the proof $\beta_{S^{\prime}}=\beta_{S}$ so that

$$
0 \leq\left(\sigma^{\prime}\right)^{2} \leq \lim _{\varepsilon \rightarrow 0} \lim _{n \rightarrow \infty} \int_{|x|<\varepsilon} x^{2} d \Lambda_{n}(x)=0
$$

so that $3^{0}$ holds with $\left(\sigma^{\prime}\right)^{2}=0$, and consequently $S^{\prime}$ converges to $X \sim\left(\alpha^{\prime}, 0, \Lambda\right)$. We are thus left to consider the case when $\beta_{S}=2$ (recall (3.1) implies $\left.\beta_{S} \leq 2\right)$. Then we assume $E\left(Y^{2}\right)<\infty$ and as before we have 


$$
\begin{aligned}
& \sigma_{n}^{2}(\varepsilon)=\int_{|x|<\varepsilon} x^{2} \int_{|u x|<\varepsilon} u^{2} d F_{Y}(u) d M_{n}(x) \\
& +\int_{|x| \geq \varepsilon} x^{x^{2}} \int_{|u x|<\varepsilon} u^{2} d F_{Y}(u) d M_{n}(x)-\Sigma\left(\int_{|x|<\varepsilon}{ }^{x} \int_{|u x|<\varepsilon} u d F_{Y}(u) d F_{n j}(x)\right)^{2} \\
& -\Sigma\left(\int_{|x| \geq \varepsilon} 2^{x} \int_{|u x|<\varepsilon} u d F_{Y}(u) d F_{n j}(x)\right)^{2} \\
& -2 \Sigma\left(\int_{|x|<\varepsilon} 2^{x} \int_{|u x|<\varepsilon} u d F_{Y}(u) d F_{n j}(x)\right)\left(\int_{|x| \geq \varepsilon} x \int_{|u x|<\varepsilon} u d F_{Y}(u) d F_{n j}(x)\right) .
\end{aligned}
$$

Applying (3.10) to the second term, Claim 2 to the last term and Claim 1 to the 4 th term on the right hand side of the last equality in (3.13) and upon adding and subtracting

$$
\Sigma\left(\int_{|x|<\varepsilon^{2}} \mathbf{x} \int_{|u|<1 / \varepsilon} u d F_{Y}(u) d F_{n j}(x)\right)^{2}
$$

we have

$$
\begin{aligned}
\sigma_{n}^{2}(\varepsilon)= & \int_{|x|<\varepsilon} x^{2} \int_{|u x|<\varepsilon} u^{2} d F_{Y}(u) d M_{n}(x) \\
& -\left(\int_{|u|<1 / \varepsilon} u d F_{Y}(u)\right)^{2} \Sigma\left(\int_{|x|<\varepsilon^{2}} x d F_{n j}(x)\right)^{2} \\
+ & \Sigma\left\{\left(\int_{|x|<\varepsilon} \int_{|u|<1 / \varepsilon} u_{Y}(u) d F_{n j}(x)\right)^{2}\right. \\
& \left.-\left(\int_{|x|<\varepsilon}{ }^{2} \int_{|u x|<\varepsilon} u d F_{Y}(u) d F_{n j}(x)\right)^{2}\right\}+g_{1}(n, \varepsilon)
\end{aligned}
$$


where $\lim _{\varepsilon \rightarrow 0} \lim _{n \rightarrow \infty} \sup _{1}(n, \varepsilon)=0$.

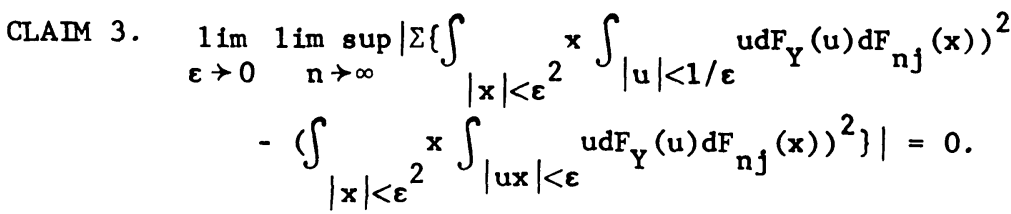

PROOF. Using the equality $a^{2}-b^{2}=(a-b)(a+b)$ we have

$$
\begin{aligned}
& \mid\left(\int_{|x|<\varepsilon} 2^{x} \int_{|u|<1 / \varepsilon} u_{Y}(u) d F_{n j}(x)\right)^{2} \\
& -\left(\int_{|x|<\varepsilon^{2}} x \int_{|u x|<\varepsilon} u d F_{Y}(u) d F_{n j}(x)\right)^{2} \mid \\
& =\left\{2 \int_{|x|<\varepsilon}{ }^{2} \int_{|u|<1 / \varepsilon} u_{Y}(u) d F_{n j}(x)\right. \\
& \left.+\int_{|x|<\varepsilon}{ }^{2} \int_{1 / \varepsilon<|u|<\varepsilon /|x|} u_{Y}(u) d F_{n j}(x)\right\}\left\{\int_{1 / \varepsilon<|u|<\varepsilon /|x|} u d F_{Y}(u) d F_{n j}(x)\right\}
\end{aligned}
$$

For simplicity we denote

$$
f_{n j}(\varepsilon)=\int_{|x|<\varepsilon^{2}} x \int_{1 / \varepsilon<|u|<\varepsilon /|x|} u d F_{Y}(u) d F_{n j}(x) .
$$

Then the right hand side of (3.15) becomes equal to

$$
2 f_{n j}(\varepsilon) \int_{|x|<\varepsilon} 2^{x} \int_{|u|<1 / \varepsilon} u d F_{Y}(u) d F_{n j}(x)+f_{n j}^{2}(\varepsilon) .
$$


Thus to complete the proof of Claim 3 we must show

$$
\lim _{\varepsilon \rightarrow 0} \underset{n \rightarrow \infty}{\lim \sup _{n \rightarrow} \sum f_{n j}^{2}(\varepsilon)=0}
$$

and

$$
\left.\lim _{\varepsilon \rightarrow 0} \lim _{n \rightarrow \infty} \sup _{n \rightarrow \infty} f_{n j}(\varepsilon) \int_{|x|<\varepsilon} x \int_{|u|<1 / \varepsilon} u d F_{Y}(u) d F_{n j}(x)\right\}=0
$$

Concerning (3.16), note that by the Schwarz inequality

$$
f_{n j}^{2}(\varepsilon) \leq \int_{|x|<\varepsilon} x^{2} \int_{|u|>1 / \varepsilon} u^{2} d F_{Y}(u) d F_{n j}(x)
$$

and letting $a\left(\varepsilon^{2}\right)=\lim _{n \rightarrow \infty} \int_{|x|<\varepsilon^{2}} x^{2} d M_{n}(x)$ we have $a\left(\varepsilon^{2}\right) \downarrow C_{0}$ as $\varepsilon \downarrow 0$ and hence

$$
\lim _{\varepsilon \rightarrow 0} \underset{n \rightarrow \infty}{1 \operatorname{im} \sup } \sum f_{n j}^{2}(\varepsilon) \leq \lim _{\varepsilon \rightarrow 0}\left\{\int_{|u|>1 / \varepsilon} u^{2} d F_{Y}(u) a\left(\varepsilon^{2}\right)\right\}=0
$$

so that (3.16) holds. In a similar manner, concerning (3.17) we have

$$
\begin{aligned}
& \left|f_{n j}(\varepsilon) \int_{|x|<\varepsilon^{2}} x \int_{|u|<1 / \varepsilon} u d F_{Y}(u) d F_{n j}(x)\right| \\
& \quad \leq\left\{\int_{|x|<\varepsilon^{2}}|x| \int_{|u|>1 / \varepsilon}|u| d F_{Y}(u) d F_{n j}(x)\right\}\left\{E|Y| \int_{|x|<\varepsilon^{2}}|x| d F_{n j}(x)\right\} \\
& \quad \leq E(Y)\left\{\int_{|u|>1 / \varepsilon}|u| d F_{Y}(u)\right\}\left\{\int_{|x|<\varepsilon^{2}} x^{2} d F_{n j}(x)\right\} .
\end{aligned}
$$


Thus $\left|\Sigma\left\{f_{n j}(\varepsilon) \int_{|x|<\varepsilon^{2}} x \int_{|u|<1 / \varepsilon} u d F_{Y}(u) d F_{n j}(x)\right\}\right|$

$\leq E|Y|\left\{\int_{|u|>1 / \varepsilon}|u| d F_{Y}(u) \int_{|x|<\varepsilon^{2}} x^{2} d M_{n}(x)\right\}$, from which (3.17) follows easily .

Now using Claim 3 in (3.14) and upon adding and subtracting

$\left(\int_{|u|<1 / \varepsilon} u d F Y(u)\right)^{2} \int_{|x|<\varepsilon} x^{2} d M_{n}(x)$, we obtain from (3.14),

$$
\begin{aligned}
\sigma_{n}^{2}(\varepsilon) & =\int_{|x|<\varepsilon} x^{2}\left\{\int_{|u|<\varepsilon /|x|} u^{2} d F_{Y}(u)-\left(\int_{|u|<1 / \varepsilon} u d F_{Y}(u)\right)^{2}\right\} d M_{n}(x) \\
& +\left(\int_{|u|<1 / \varepsilon} u d F_{Y}(u)\right)^{2}\left\{\int_{|x|<\varepsilon} x^{2} d M_{n}(x)-\Sigma\left(\int_{|x|<\varepsilon} x d F_{n j}(x)\right)^{2}\right\} \\
& +g_{2}(n, \varepsilon) \text { where } \lim _{\varepsilon \rightarrow 0} \lim _{n \rightarrow \infty} \sup _{2} g_{2}(n, \varepsilon)=0
\end{aligned}
$$

Then, if we use the inequalities

$$
\begin{aligned}
& \left\{\int_{|x|<\varepsilon} x^{\left.x^{2} d M_{n}(x)\right\}\left\{\int_{|u|<1 / \varepsilon} u^{2} d F_{Y}(u)\right\}}\right. \\
& \quad \leq \int_{|x|<\varepsilon^{2}} x^{2} \int_{|u x|<\varepsilon} u^{2} d F_{Y}(u) d M_{n}(x) \leq E\left(Y^{2}\right) \int_{|x|<\varepsilon^{2}} x^{2} d M_{n}(x)
\end{aligned}
$$

in (3.18), we obtain the following two sided bound for $\sigma_{n}^{2}(\varepsilon)$ : 


$$
\begin{aligned}
& \left\{\int_{|x|<\varepsilon} x^{2} x_{n}(x)\right\}\left\{\int_{|u|<1 / \varepsilon} u^{2} d F_{Y}(u)-\left(\int_{|u|<1 / \varepsilon} u d F_{Y}(u)^{2}\right\}+g_{2}(n, \varepsilon)\right. \\
& \quad+\left(\int_{|u|<1 / \varepsilon} u d F_{Y}(u)\right)^{2}\left\{\int_{|x|<\varepsilon^{2}} x^{2} d M_{n}(x)-\Sigma\left(\int_{|x|<\varepsilon^{2}} x d F_{n j}(x)\right)^{2}\right\} \\
& \quad \leq \sigma_{n}^{2}(\varepsilon) \leq\left\{\int_{n}|x|<\varepsilon^{2} x^{2} d M_{n}(x)\right\}\left(E\left(Y^{2}\right)-\left(\int_{|u|<1 / \varepsilon} u d F_{Y}(u)\right)^{2}\right\}+g_{2}(n, \varepsilon) \\
& \quad+\left(\int_{|u|<1 / \varepsilon} u d F_{Y}(u)\right)^{2}\left\{\int_{|x|<\varepsilon^{2}} x^{2} d M_{n}(x)-\Sigma\left(\int_{|x|<\varepsilon^{2}} x_{n j}(x)\right)^{2}\right\}
\end{aligned}
$$

Using the fact that $\lim _{\varepsilon \rightarrow 0}\left\{\begin{array}{l}\text { lim sup } \\ \text { lim inf }\end{array}\right\}_{n \rightarrow \infty}$ of the extremities in (3.19) are equal when (3.1) holds, we obtain

$$
\begin{aligned}
& C_{0} \operatorname{Var}(Y)+(E(Y))^{2} \sigma^{2} \leq \lim _{\varepsilon \rightarrow 0} \lim _{n \rightarrow \infty} \inf \sigma_{n}^{2}(\varepsilon) \\
& \quad \leq \lim _{\varepsilon \rightarrow 0} \lim _{n \rightarrow \infty} \sup _{n}^{2}(\varepsilon) \leq C_{0} \operatorname{Var}(Y)+(E(Y))^{2} \sigma^{2}
\end{aligned}
$$

where $\mathrm{C}_{0}$ is the constant given in (3.1), so that $\left(\sigma^{\prime}\right)^{2}$ exists and is given by $\left(\sigma^{\prime}\right)^{2}=C_{0} \operatorname{Var}(Y)+(E(Y))^{2} \sigma^{2}$.

9) In this part of the proof we show that if $S^{\prime}$ is convergent (so that $\lim _{\varepsilon \rightarrow 0} \lim _{\mathrm{n} \rightarrow \infty} \sup _{\mathrm{n}}^{2}(\varepsilon)=\left(\sigma^{\prime}\right)^{2}=\underset{\varepsilon \rightarrow 0}{\lim } \lim _{\mathrm{n} \rightarrow \infty} \sigma_{\mathrm{n}}^{2}(\varepsilon)$ ) then necessarily (3.1) holds. By part 7) we know that we may assume $\lim _{n \rightarrow \infty} \int_{|x|<\varepsilon} x^{2} d M_{n}(x)<\infty$ so that all of the previous equations leading up to (3.19) still remain valid. Let us then subtract the quantity 


$$
\left(\int_{|x|<1 / \varepsilon} \operatorname{udF}_{Y}(u)\right)^{2}\left\{\int_{|x|<\varepsilon} x^{2} x_{n}(x)-\Sigma\left(\int_{|x|<\varepsilon} 2^{x d F}{ }_{n j}(x)\right)^{2}\right\}+g_{2}(n, \varepsilon)
$$

throughout the inequality (3.19) to obtain

$$
\begin{aligned}
& \left\{\int_{|x|<\varepsilon} x^{2} d M_{n}(x)\right\}\left\{\int_{|x|<1 / \varepsilon} u^{2} d F_{Y}(u)-\left(\int_{|u|<1 / \varepsilon} u d F_{Y}(u)\right)^{2}\right\} \\
& \leq \sigma_{n}^{2}(\varepsilon)-\left(\int_{|x|<1 / \varepsilon} x d F_{Y}(x)\right)^{2}\left[\int_{|x|<\varepsilon} 2^{x^{2} d M_{n}(x)}\right. \\
& \left.-\Sigma\left(\int_{|x|<\varepsilon} 2^{2 d F} n j(x)\right)^{2}\right\}-g_{2}(n, \varepsilon) \\
& \leq\left\{\int_{|x|<\varepsilon} 2^{2} \mathrm{xM}_{\mathrm{n}}(\mathrm{x})\right\}\left\{\mathrm{E}\left(\mathrm{Y}^{2}\right)-\left(\int_{|u|<1 / \varepsilon} \mathrm{udF}_{\mathrm{Y}}(\mathrm{u})\right)^{2}\right\} .
\end{aligned}
$$

Now, $\lim _{\varepsilon \rightarrow 0}\left\{\begin{array}{ll}\lim \text { sup } \\ \lim \text { inf }\end{array}\right\}_{n \rightarrow \infty}$ of the inside of $(3.20)$ equals $\left(\sigma^{\prime}\right)^{2}-(E(Y))^{2} \sigma^{2}$ while on the outside of (3.20) the above limits yield $\operatorname{Var}(Y) \lim _{\varepsilon \rightarrow 0}\left\{\begin{array}{l}\lim \sup \\ \lim \inf \end{array}\right\}_{n \rightarrow \infty} \int_{|x|<\varepsilon^{2}}|x|^{2} \mathrm{dM}_{\mathrm{n}}(\mathrm{x})$. We thus conclude (3.1) holds with $\operatorname{Var}(\mathrm{Y}) \mathrm{C}_{0}=\left(\sigma^{\prime}\right)^{2}-(\mathrm{E}(\mathrm{Y}))^{2} \sigma^{2}$. This completes the proof of the theorem.

Let us now turn to the framework of the more classical central limit theorem. If $\left\{\mathrm{x}_{1}, \mathrm{x}_{2}, \ldots\right\}$ is a sequence of independent identically distributed random variables with common distribution function $F$, we shall write $x_{1} \in D(\alpha)$ to denote the fact that $F$ (or $x_{1}$ ) is in the domain of attraction of a stable law with characteristic exponent $\alpha$. That is, 
there exists norming constants $\left\{B_{n}, n=1,2, \ldots\right\}$ and centering constants $\left\{A_{n}, n=1,2, \ldots\right\}$ such that $\sum_{j=1}^{n} X_{j} / B_{n}-A_{n}$ converges in 1 aw to a distribution which is stable with exponent $\alpha \leq 2$.

In this case the relation between the scale mixed system $S^{\prime}$ and the original system $S$ takes on a particularly appealing form. Our condition for convergence only depends upon the moments of $Y$ and the index $\alpha$.

THEOREM 4. Let $\left\{X_{1}, Y_{1}, X_{2}, Y_{2}, \ldots\right\}$ be independent random variables, $X_{n}$ having distribution function $F_{X}$ and $Y_{n}$ having distribution function $\mathrm{F}_{\mathrm{Y}}$

1) If $E|Y|^{2}<\infty$, then $X \in D(2)$ implies $Y X \in D(2)$ and the same norming constants work. Conversely if $Y X \in D(2)$ then $X \in D(2)$ and the same norming constants work.

2) If $\mathrm{E}|\mathrm{Y}|^{\alpha+\delta}<\infty$ for some $\delta>0$, then $\mathrm{X} \in D(\alpha)$ implies $\mathrm{YX} \in D(\alpha)$ and the same norming constants work.

PROOF. The direct statement in 1) and 2) will follow from Theorem 3 once we show that (3.1) holds. Let $s=\left\{\left\{x_{j} / B_{n}, j=1,2, \ldots, n\right\}\right\}$ then as proven in [4], $X \in D(\alpha)$ implies $\beta_{S}=\alpha$. For $\alpha<2$ we have $(3.1)$ holding with $\mathrm{C}_{0}=0$ by $(2.4)$. For $\alpha=2$ we know (see Feller (1971), [6] pg. 314) that necessarily

$$
\frac{\mathrm{n}}{\mathrm{B}_{\mathrm{n}}^{2}} \int_{|\mathrm{y}|<\mathrm{B}_{\mathrm{n}}} \mathrm{y}^{2} \mathrm{dF} \mathrm{X}(\mathrm{y}) \rightarrow \mathrm{C}_{0} \text { as } \mathrm{n} \rightarrow \infty
$$


Using the fact that $\frac{n}{B_{n}^{2}} \int_{|y|<B_{n} \varepsilon} y^{2} d F_{X}(y)=\int_{|x|<\varepsilon} y^{2} d M_{n}(y)$ and that $\int_{|y|<t} y^{2} d F(y)$ is a slowly varying function of $t$ yields (3.1) in this case also. Applying Theorem 3 we obtain the convergence of $S^{\prime}=\left\{\left\{X_{j} Y_{j} / B_{n}, j=1,2, \ldots, n\right\}\right\}$. If $\alpha=2$ then $M \equiv 0$ so by $2^{0}$ of Theorem 3 , $\Lambda \equiv 0$ and $S^{\prime}$ converges to a normal distribution. If $\alpha<2$, then by $3^{0}$ of Theorem $3\left(\sigma^{\prime}\right)^{2}=0$ and with

$$
M(x)=\left\{\begin{array}{ccc}
C_{1}|x|^{-\alpha} & \text { if } & x<0 \\
-C_{2} x^{-\alpha} & \text { if } & x>0
\end{array}\right.
$$

we have by $2^{0}$ of Theorem 3

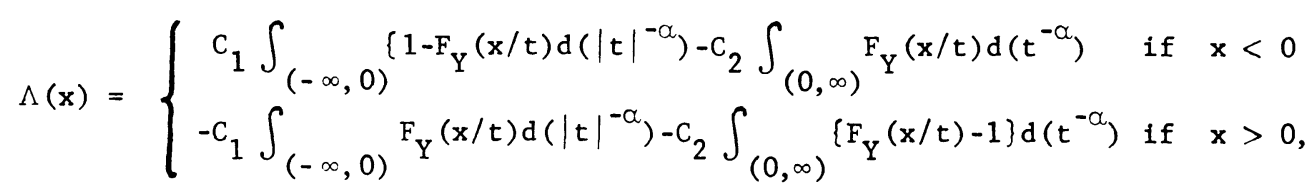

or equivalently, upon integrating by parts

$$
\Lambda(x)=\left\{\begin{array}{l}
C_{1} \int_{(-\infty, 0)}|t|^{-\alpha} d_{Y}\left(\frac{x}{t}\right)+c_{2} \int_{(0, \infty)} t^{-\alpha} d F_{Y}\left(\frac{x}{t}\right) \text { if } x<0 \\
C_{1} \int_{(-\infty, 0)}|t|^{-\alpha} d_{Y}\left(\frac{x}{t}\right)+c_{2} \int_{(0, \infty)} t^{-\alpha} d F_{Y}\left(\frac{x}{t}\right) \text { if } x>0 .
\end{array}\right.
$$

Upon simplifying (3.23) we obtain

$$
\Lambda(x)=\left\{\begin{array}{lll}
a_{1}|x|^{-\alpha} & \text { if } & x<0 \\
-a_{2} x^{-\alpha} & \text { if } & x>0
\end{array}\right.
$$


i.e., $\mathrm{X}_{\mathrm{n}} \mathrm{Y}_{\mathrm{n}}$ is in the domain of attraction of a stable law with characteristic exponent $\alpha$, and the same norming constants work.

Let us suppose now that $\mathrm{YX} \in \mathcal{D}(2)$ and $E|\mathrm{Y}|^{2}<\infty$. We shall use the general limit theorem to show $\sum_{j=1}^{n} X_{j} / B_{n}-A_{n}$ converges to a normal distribution. With $M_{n}$ given by (2.1) and $\Lambda_{n}$ given by (3.3) we shall show $\Lambda_{n}(x) \rightarrow 0$ for $x \neq 0$ implies $M_{n}(x) \rightarrow 0$ for $x \neq 0$. Indeed let $t$ be such that $P[|\mathrm{Y}|>\mathrm{t}]>0$. Then

$$
\begin{aligned}
& 0 \leftarrow \int_{|x|>t^{2}} d \Lambda_{n}(x)=\iint_{|u x|>t^{2}} d F_{Y}(u) d M_{n}(x) \\
& \quad \int_{|x|>t} \int_{|u x|>t}{ }^{2} d F_{Y}(u) d M_{n}(x) \geq \int_{|x|>t} P[|Y|>t] d M_{n}(x) .
\end{aligned}
$$

Since $P[|Y|>t]>0, \int_{|x|>t} d_{n}(x) \rightarrow 0$, thus the 1imiting Levy function is 0 .

Let us complete the proof by showing

$$
\lim _{\varepsilon \downarrow 0}\left\{\lim \sup _{1 \text { im } \inf }\right\}_{n \rightarrow \infty} \int_{|x|<\varepsilon} x^{2} d M_{n}(x)-n\left(\int_{|x|<\varepsilon} x d F(x)\right)^{2}
$$

exists and is finite. In view of part 7) of the proof of Theorem 3 we know we must have

$$
C_{0}=\lim _{\varepsilon \rightarrow 0} \lim _{n \rightarrow \infty} \int_{|x|<\varepsilon} x^{2} d M(x)<\infty .
$$


Utilizing the inequality (3.20) of Theorem 3 we see that we must only show for

$$
\mathrm{C}_{1}=\lim _{\varepsilon \downarrow 0} \lim _{\mathrm{n} \rightarrow \infty} \int_{|\mathrm{x}|<\varepsilon} \mathrm{x}^{2} \mathrm{dM} \mathrm{n}(\mathrm{x})
$$

we have $\mathrm{C}_{0}=\mathrm{C}_{1}$. Now by Fatou's lemma and by $(3.21)$

$$
\begin{aligned}
C_{2} & \equiv \lim _{n \rightarrow \infty} \int_{|x|<\varepsilon} x^{2} d \Lambda_{n}(x)=\lim _{n \rightarrow \infty} \inf \iint_{|u x|<\varepsilon} x^{2} u^{2} d M_{n}(x) d F_{Y} u \\
& \leq \lim _{\varepsilon \downarrow 0} \int \lim _{n \rightarrow \infty} \inf ^{2} \int_{|u x|<\varepsilon} x^{2} d M_{n}(x) d F_{Y}(u)=C_{1} E\left(Y^{2}\right) .
\end{aligned}
$$

On the other hand for $\eta>0$ choose $\delta$ so small that $\int_{|u| \geq \delta} u^{2} d F_{Y}(u)>$ $(1-\eta) E\left(Y^{2}\right)$. Then $C_{2} \geq \lim _{n \rightarrow \infty} \sup _{|u| \geq 0} \int^{2} \int_{|x|<\varepsilon / \delta} x^{2} d M_{n}(x) d F_{Y}(u) \geq$ $(1-\eta) E\left(Y^{2}\right) 1 \operatorname{im}_{n \rightarrow \infty} \int_{|x|<\varepsilon / \delta} x^{2} d M_{n}(x)$. Thus

$$
(1-\eta) E\left(Y^{2}\right) C_{0} \leq C_{2} \leq E\left(Y^{2}\right) C_{1} \leq E\left(Y^{2}\right) C_{0}
$$

Since $\eta>0$ is arbitrary $C_{1}=C_{0}$ and $\sum_{j=1}^{n} x_{j} / B_{n}-A_{n}$ converges to a normal distribution.

REMARK 2. i) The first part of Theorem 4 should be compared to a result of $\mathrm{H}$. Tucker (1968) who considered sums of random variables in the domain of attraction of the stable distributions instead of their products. He shows that if $\mathrm{EY}^{2}<\infty$ and $X \in D(\alpha)$ then $X+Y \in D(\alpha)$ and the same norming constants work. If $X+Y \in D(\alpha)$ and $E(Y)^{2}<\infty$, then a 
slight modification of his methods yields $X \in D(\alpha)$. Combining our result with his we find that if $\mathrm{X}, \mathrm{Y}, \varepsilon$ are independent random variables, $\mathrm{EY}^{2}<\infty$ and $\mathrm{E} \varepsilon^{2}<\infty$, then $\mathrm{X} \in \mathcal{D}(\alpha) \Rightarrow \mathrm{YX}+\varepsilon \in D(\alpha)$ and the same norming constants work. For $\alpha=2, X \in D(2) \Leftrightarrow Y X+\varepsilon \in D(2)$ with the same norming constants.

ii) In Theorem 4 we can actually calculate the scale change involved in the distribution of the limit laws of $S$ and $S^{\prime}$ when $a<2$. Namely, in going from (3.23) to (3.24) we have

$$
\Lambda(x)= \begin{cases}a_{1}|x|^{-\alpha} & x<0 \\ -a_{2} x^{-\alpha} & x>0\end{cases}
$$

where $a_{1}=c_{1} \int_{(0, \infty)} t^{\alpha} d_{Y}(t)+c_{2} \int_{(-\infty, 0)}|t|^{\alpha} d F_{Y}(t)$ and $a_{2}=c_{2} \int_{(0, \infty)} t^{\alpha} \mathrm{dF}_{Y}(t)+c_{1} \int_{(-\infty, 0)}|t|^{\alpha} d_{Y}(t)$. This follows immediately from (3.23) by the change of variables $z=x t^{-1}$.

iii) It can be shown that for $\alpha<2, \quad Y X \in D(\alpha)$ and $E|Y|^{\alpha+\eta}<\infty$ implies that $E|X|^{\alpha-\delta}<\infty$ and $E|X|^{\alpha+\delta}=\infty$ for all $\delta$, hence $\beta_{S}=\alpha$. I suspect that in fact $X \in D(\alpha)$ however, I have been unable to establish this for $\alpha \neq 2$.

iv) Suppose that

$$
\lim _{\varepsilon \rightarrow 0}\left\{\begin{array}{l}
\lim \sup \\
\lim \text { inf }
\end{array}\right\}_{n \rightarrow \infty} \int_{|x|<\varepsilon}|x|^{\beta} S_{d M_{n}}(x)=C_{0}
$$


then for $1-F_{Y}(x)+F_{Y}(-x) \sim \frac{C_{1}}{|x|^{\beta}}$ we have $\Lambda_{n}(x) \rightarrow \frac{C_{0} C_{1}}{|x|^{\beta_{S}}}+\Lambda(x)$ where $\Lambda(x)$ is given by $2^{0}$ of Theorem 3 . This is a Levy spectral function when $\beta_{S}<2$. Thus we see that some random scale changes introduce a stable component into the limit 1 aw.

We can also use Theorem 4 to derive some interesting statements about slowly varying functions which would be difficult to prove by other means. The precise formulations are given in the following corollary.

COROLLARY $1^{0}$. Suppose $\mathrm{EY}^{2}<\infty$. Then $\int_{|x|<t} \mathrm{x}^{2} \mathrm{dF} X(\mathrm{x})$ is a slowly varying function of $t$ if and only if $\iint_{|u x|<t} x^{2} u^{2} d F_{X}(x) d F_{Y}(u)$ is $a$ slowly varying function of $t$.

$2^{0}$. Suppose

a) $\int_{|x|<t} x^{2} d F_{X}(x)$ varies regularly with exponent $2-\alpha$ as a function of $t$ and also

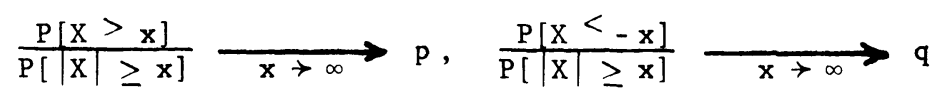

b) and $\mathrm{E}|\mathrm{Y}|^{\alpha+\delta}<\infty$ for some $\delta>0$, then

$$
\int_{(-\infty, \infty)} \int_{|u x|<t} x^{2} u^{2} d F_{Y}(u) d F_{X}(x)
$$

varies regularly with exponent $2-\alpha$ as a function of $t$ and also 


$$
\begin{aligned}
& \frac{\int_{(-\infty, \infty)} P[t Y \geq x] d F_{X}(t)}{\int_{(-\infty, \infty)} P[|t Y| \geq x] d F_{X}(t)} \underset{x \rightarrow \infty}{P^{\prime},} \\
& \frac{\int_{(-\infty, \infty)} P[t Y \leq-x] d F_{X}(t)}{\int_{(-\infty, \infty)} P[|t Y| \geq x] d F_{X}(t)} \underset{x \rightarrow \infty}{\longrightarrow} q^{\prime}
\end{aligned}
$$

for some $\mathrm{p}^{\prime}, \mathrm{q}^{\prime} \geq 0$ with $\mathrm{p}^{\prime}+\mathrm{q}^{\prime}>0$.

PROOF. This follows immediately from Theorem 4 by utilizing the necessary and sufficient conditions given in Feller (1971) for a distribution to belong to the domain of attraction of a stable law.

In the previous theorem we observed an interesting phenomenon. Namely we took an infinitesimal system $S$ and subjected it to an arbitrary random scale change with $\mathrm{E}|\mathrm{Y}|^{\beta} \mathrm{S}^{+\delta}<\infty$ and we obtained a new system $S^{\prime}$ which was convergent. Moreover the limit distributions of $\mathrm{S}$ and $\mathrm{S}^{\prime}$ were of the same type. In problems where $x_{n i}$ represents a "true" or theoretical measurement of some occurrence and $Y_{i}$ the scale change in the measuring device used to measure the occurrence, we obtain as an observation the product $\mathrm{X}_{n i} \mathrm{Y}_{i}$. It is of interest to determine when limit distribution calculated from the empirical data $\left\{\left\{x_{n j}{ }_{j}, j=1,2, \ldots, k_{n}\right\}\right\}$ is of the same type as that from $\left\{\left\{x_{n j}, j=1,2, \ldots, k_{n}\right\}\right\}$. For normed sums in the domain of attraction of a stable 1aw, Theorem 4 answers the question and Remark 2ii) allows 
us to calculate the scale change. In general the following theorem tells us that in non-stable limits the empirical data may yield a different type distribution than the theoretical data $\left\{\left\{x_{n j}, j=1,2, \ldots, k_{n}\right\}\right\}$.

THEOREM 5. In order that a limit distribution be preserved in type under all random scale mixtures with $\mathrm{E}|\mathrm{Y}|^{\beta^{+\delta}}<\infty$ it is necessary and sufficient that the limit distribution type be either purely stable or a mixture of stable and normal.

PROOF. The sufficiency follows from $2^{0}$ and $3^{0}$ of Theorem 3 of Theorem 3 as we calculated in Theorem 4, and in fact the scale change involved is given in Remark $2 i i)$.

Suppose now that the limit distribution is preserved in type when subject to random scale change. Then with $S$ and $S^{\prime}$ as defined in Theorem 3 we know $Z \sim\left(\gamma^{\prime},\left(\sigma^{\prime}\right)^{2}, \Lambda\right)$ is of the same type as $X \sim\left(\gamma, \sigma^{2}, M\right)$, thus $\left(\sigma^{\prime}\right)^{2}=a^{2} \sigma^{2}$ and $\Lambda(x)=M(x / a)$ for some constant a. As in 2$)$ of the proof of Theurem 3 we know that

$$
\Lambda(x)= \begin{cases}\int_{-\infty}^{\infty}|M(x / t)| d F_{Y}(t) & x<0 \\ -\int_{-\infty}^{\infty}|M(x / t)| d F_{Y}(t) & x>0\end{cases}
$$

If $M(x) \equiv 0$, then both $X$ and $Z$ are normally distributed. If $M(x) \not \equiv$, then we must show $M$ is given by (3.22) of Theorem 4. Using the fact that $\Lambda(x)=M(x / a)$ in (3.25) and letting

$$
h(t)=\frac{|M(x / t)|}{M(x / a)} \text {, we see that } h \text { is a function of } t
$$


alone, the equality holding a.e. $\left[\mathrm{dF}_{\mathrm{Y}}\right]$. Since $\mathrm{Y}$ could be chosen to be absolutely continuous we have (3.26) holding on a dense set of points $t$. For simplicity in calculation we shall consider the case $x>0, t>0$, a $>0$ and denote $N(x)=M(x) / M(1)$. The other cases may be considered similarly. Rewriting the equation (3.26) yields

$$
N(x / a) h(t)=N(x / t)
$$

and hence for $x=a$

$$
h(a / y)=\frac{N(x y / a)}{N(x / a)}=N(y)
$$

Thus

$$
N\left(y^{2}\right)=N(y) h(a / y)=(N(y))^{2}
$$

and by induction for any $k$

$$
N\left(y^{k}\right)=N\left(y^{k-1}\right) h(a / y)=N^{k}(y) .
$$

Letting $U(x)=-\ln \left|N\left(e^{x}\right)\right|$, the above equation becomes

$1 / k U(k \ln x)=U(\ln x)$ or

$1 / k U(k y)=U(y)$. By Lemma 3, page 277 of Feller (1971), this implies $U(x)=x^{\rho}$ or equivalently $N(x)=x^{\rho}$. That $-2<\rho<0$ follows from $M$ being 
a Levy Spectral function. Thus $M(x)=\frac{M(1)}{x^{\alpha}}$ for $x>0$. Similarly we can show $M(x)=\frac{M(-1)}{|x|^{\beta}}$ for $x<0$. We see that $\alpha=\beta$ since the 1imit distribution is of the same type whether multiplied by $\mathrm{Y} \leq 0$ or $\mathrm{Y} \geq 0$. The result then follows easily from the calculation of $\Lambda(x)$ in both cases. This completes the proof of the theorem.

REMARK. The problem considered here could have been solved by defining the class $\mathrm{C}_{S}$ of random variables by

$$
C_{S}=\left\{Y: \lim _{\varepsilon \rightarrow 0} \lim _{n \rightarrow \infty} \sup \left\{\int_{|x|>\varepsilon}\left\{1-F_{Y}(x t)+F_{Y}(-x t)\right\} d M_{n}(x)=0\right\}\right\},
$$

and proving the main theorem for members of this class. Some such account must be made of the complex interaction of $M_{n}(t)$ as $(t, n) \rightarrow(0, \infty)$ and $1-F_{Y}(t)$ as $t \rightarrow \infty$. Since moment conditions are perhaps a more natural approach, we choose to introduce the index $\beta_{S}$ instead. To avoid the borderline case $1-F_{Y}(y) \sim y^{-\beta} S$, we choose to assume $E|Y|^{\beta}{ }^{+\delta}<\infty$.

\section{ACKNOWLEDGMENT}

This paper is based in part upon results obtained in the author's doctoral dissertation completed in 1975 at the University of California, Irvine, under the direction of Professor Howard G. Tucker. I would like to thank Professor Tucker for posing the problem solved here, and constantly expressing an interest in it. I benefited considerably from conversations with him. 


\section{REFERENCES}

1. Berman, S. M. Sign-Invariant Random Variables and Stochastic Processes with Sign Invariant Increments, Trans. Amer. Math. Soc. 119 (1965) 216-243, MR 23 A689.

2. Blumentha1, R. M. and Getoor, R.K. Sample Functions of Stochastic Processes with Stationary Independent Increments, J. Math. Mech. 10 (1961) 493-516, MR 23, 非A689.

3. Brockett, P. L. Limit Distributions of Sums under Mixing of the Scale Parameter, Ph.D. dissertation, University of California, Irvine, 1975.

4. Brockett, P. L. Variational Sums of Infinitesimal Systems, $\underline{Z}$. Wahr. 38 (1977) 293-307.

5. Brockett, P. L. and Hudson, W. N. Variational Path Properties of Additive Processes, unpublished manuscript.

6. Feller, W. An Introduction to Probability Theory and its App1ications 2, 2nd Ed. John Wiley and Sons, Inc., New York, 1971.

7. Gnedenko, B. V. and Kolmogorov, A. N. Limit Distributions for Sums of Independent Random Variables, Addison-Wesley, Cambridge, Mass ., 1968.

8. Tucker, H. G. A Graduate Course in Probability, Academic Press, New York and London, 1967, MR 36 非4593.

9. Tucker, H. G. Convolutions of Distributions Attracted to Stable Laws, Ann. Math. Statist. 39 (1968) 1381-1390. 


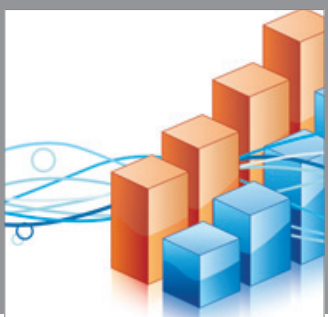

Advances in

Operations Research

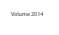

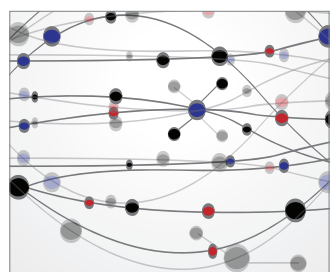

\section{The Scientific} World Journal
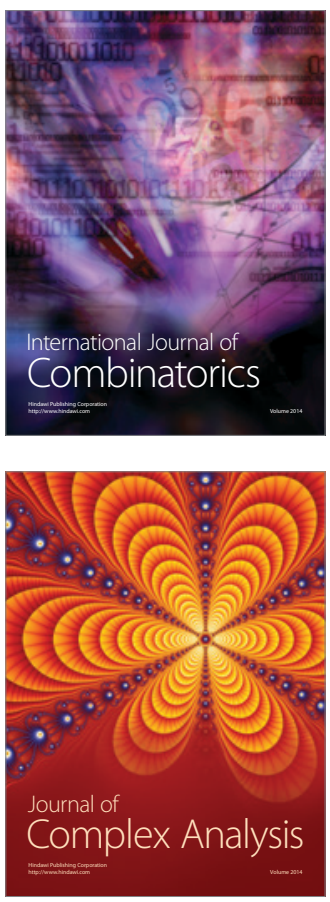

International Journal of

Mathematics and

Mathematical

Sciences
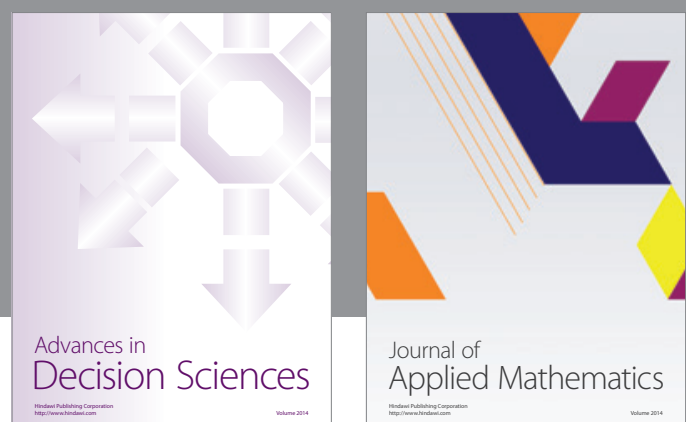

Journal of

Applied Mathematics
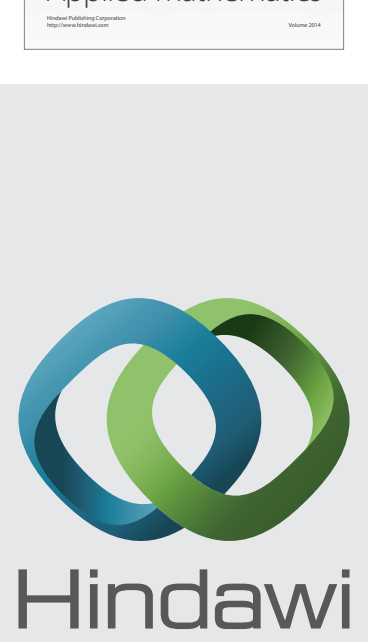

Submit your manuscripts at http://www.hindawi.com
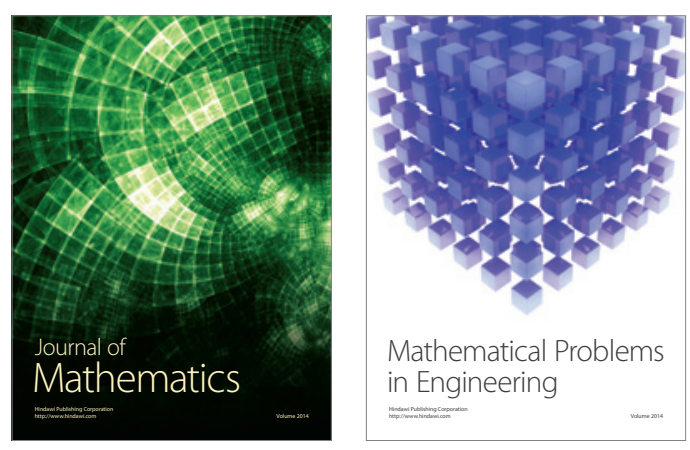

Mathematical Problems in Engineering
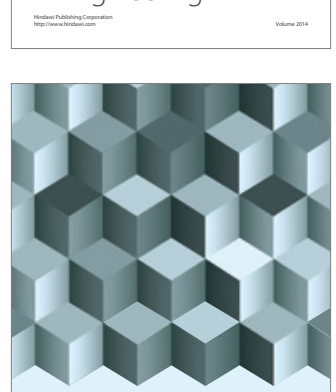

Journal of

Function Spaces
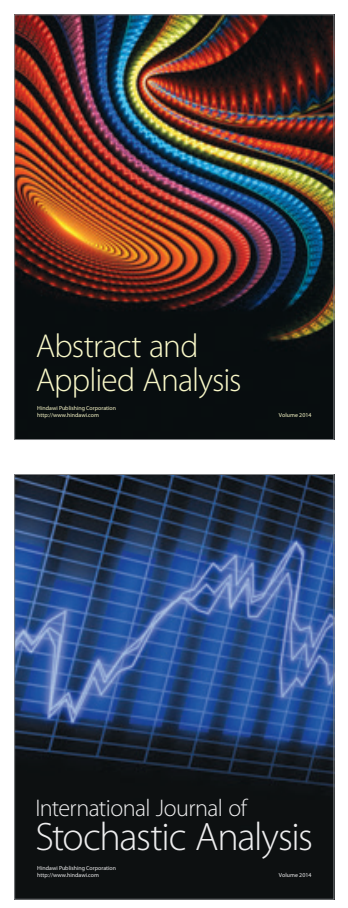

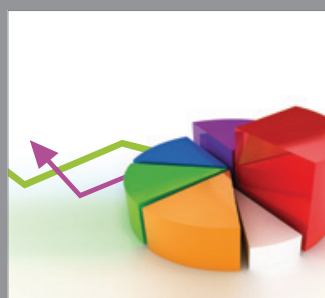

ournal of

Probability and Statistics

Promensencen
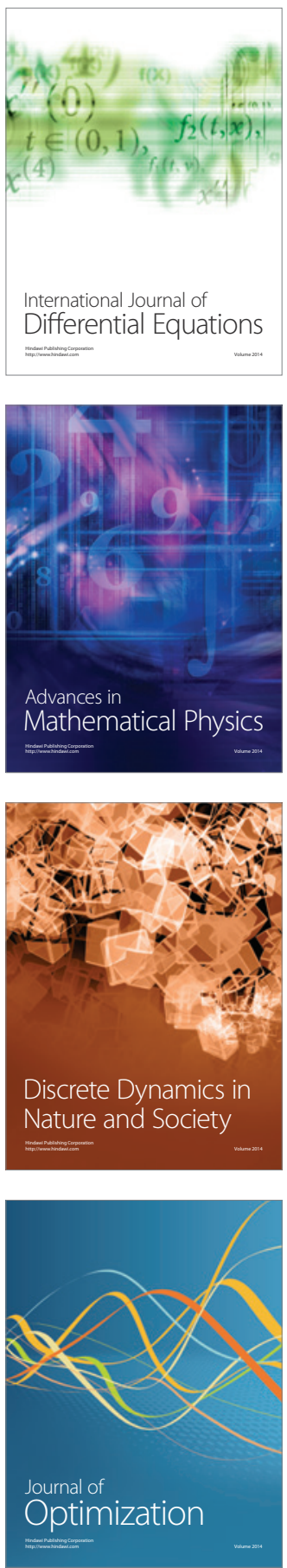